\title{
ANÁLISE DO FATOR DE COMPRESSIBILIDADE NO EQUILÍBRIO LÍQUIDO-VAPOR DE FLUIDOS DE PETRÓLEO UTILIZANDO O SIMULADOR PVTpetro
}

\author{
A. M. BARBOSA NETO ${ }^{1,2}$, L. A. A. P. FONSECA ${ }^{2}$, E. C. RYLO ${ }^{2}$ e A. C. BANNWART ${ }^{1}$ \\ ${ }^{1}$ Universidade Estadual de Campinas, Faculdade de Engenharia Mecânica, Departamento de \\ Energia - Divisão de Petróleo \\ ${ }^{2}$ SimWorx - Engenharia, Pesquisa \& Desenvolvimento \\ E-mail para contato: aneto@dep.fem.unicamp.br
}

\begin{abstract}
RESUMO - Na exploração de petróleo, fluidos são produzidos desde o reservatório até a planta de processamento. Ao longo deste sistema, o fluido de petróleo escoa sob diferentes condições de pressão e temperatura acarretando mudanças no comportamento de fases da mistura de hidrocarbonetos, podendo ocorrer a formação de duas ou mais fases em equilíbrio. Os métodos de cálculo para o fator de compressibilidade das fases em equilíbrio para um sistema óleogás, disponíveis no simulador PVTpetro, foram utilizados para avaliar o comportamento desta propriedade. Os resultados mostraram uma boa concordância entre os métodos de Cardano e numérico. Além disso, verificou-se que o comportamento do fator de compressibilidade das fases é fortemente dependente da pressão e temperatura do sistema.
\end{abstract}

\section{INTRODUÇÃO}

Nos últimos anos, as descobertas de reservatórios de petróleo portadores de óleo leve, de gás e condensado têm aumentado em campos marítimos brasileiros (ANP, 2014). A predição do comportamento termodinâmico de fases é essencial para o desenvolvimento e otimização da produção de hidrocarbonetos em aplicações na engenharia de petróleo. Projetos bem sucedidos nesta área, tais como, gerenciamento da produção de um reservatório, análise de escoamento multifásico em sistemas de produção e dimensionamento de plantas de processamento de fluidos dependem da predição correta do comportamento termodinâmico de fases (Barbosa Neto et al, 2014).

Um modelo termodinâmico fornece a relação necessária entre as propriedades termodinâmicas e pode ser utilizado em combinação com relações fundamentais para gerar as propriedades requeridas para realizar os cálculos de equilíbrio de fases (Saber, 2011). Dentre estas propriedades, o fator de compressibilidade é de fundamental importância para a análise do comportamento de fases dos fluidos de petróleo. Neste sentido, as equações de estado cúbicas, tal como Peng-Robinson (1978), são possivelmente as equações mais frequentemente utilizadas para aplicações práticas na indústria de petróleo (Saber e Shaw, 2008).

Diante deste contexto, o presente trabalho teve como objetivo apresentar um estudo comparativo entre os métodos analítico e numérico da solução da Equação de Estado Cúbica 


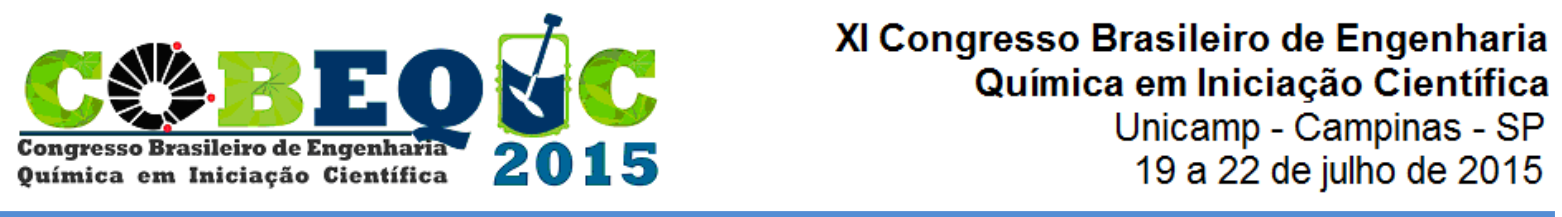

(EEC) de Peng-Robinson expresso em termos do fator de compressibilidade $Z$ para uma mistura de hidrocarbonetos utilizando o simulador PVTpetro.

\section{METODOLOGIA}

A composição do fluido de petróleo utilizado para avaliar o fator de compressibilidade das fases é descrita em Barbosa Neto, (2015). A mistura de hidrocarbonetos escolhida foi caracterizada utilizando o simulador PVTpetro (Barbosa Neto, 2015). Esta ferramenta possui um módulo de cálculo flash $(\mathrm{P}, \mathrm{T})$ isotérmico capaz de descrever as fases em equilíbrio, inclusive o fator de compressibilidade de cada fase. Para o presente trabalho, a equação de estado de Peng-Robinson, cúbica em $Z$, juntamente com a regra de mistura tipo um fluido de van der Waals foi selecionada no PVTpetro. Além disso, o simulador disponibiliza dois métodos para resolução da EEC: método de Cardano e método numérico (Newton-Raphson).

\subsection{Equação de Estado de Peng-Robinson}

A equação de Peng-Robinson (1978) é apresenta na Equação 1.

$$
p=\frac{R T}{V-b}-\frac{a \alpha(T)}{V(V+b) b(V-b)}
$$

O parâmetro $\alpha$ é uma função da temperatura e do fator acêntrico $(\omega)$ de cada componente da mistura, $a$ e $b$ são respectivamente, parâmetro de energia e covolume das moléculas. Reescrevendo a Equação $1 \mathrm{em}$ termos do fator de compressibilidade $Z$, tem-se (Ahmed, 2007):

$$
Z^{3}+(B-1) Z^{2}+\left(A-3 B^{2}-2 B\right) Z-\left(A B-B^{2}-B^{3}\right)=0
$$

na qual, $A$ e $B$ são constantes definidas para a equação de estado. Definindo os coeficientes da Equação 2:

$$
C_{1}=1 \quad C_{2}=B-1 \quad C_{3}=A-3 B^{2}-2 B \quad C_{4}=A B-B^{2}-B^{3}
$$

\subsection{Método Numérico}

A função objetivo $(f)$ que representa o método numérico é dada por:

$$
f=C_{1} Z^{3}+C_{2} Z^{2}+C_{3} Z+C_{4}
$$

e sua derivada analítica $\left(f^{\prime}\right)$ é representada pela Equação 5:

$$
f^{\prime}=3 C_{1} Z^{2}+2 C_{2} Z+C_{3}
$$

O valor de $Z$ igual a 0,5 foi estabelecido como chute inicial para o método de NewtonRaphson. A tolerância adotada como critério de convergência foi de $10^{-6}$. Após a determinação da primeira raiz da equação cúbica $\left(Z_{1}\right)$, aplica-se a abordagem analítica utilizando-se a regra de Briot-Ruffini para converter a Equação 4 em um polinômio de grau 2, conforme mostra a Equação 6: 


$$
Z^{2}+\left(Z_{1}+C_{2}\right) Z+\left[Z_{1}\left(Z_{1}+C_{2}\right)+C_{3}\right]=0
$$

A Equação 6 é resolvida pelo método convencional de Bháskara. Sendo assim, o discriminante é definido por:

$$
\text { Disc }=\left(Z_{1}+C_{2}\right)^{2}-4\left[Z_{1}\left(Z_{1}+C_{2}\right)+C_{3}\right]
$$

As soluções da Equação 6 são da forma:

$$
Z=\frac{-\left(Z_{1}+C_{2}\right) \pm \sqrt{\text { Disc }}}{2}
$$

Algumas considerações devem ser feitas durante a resolução para o discriminante deste método: Disc $<0$ : apenas uma raiz real que corresponde a raiz da fase analisada; $D i s c=0$ : tem-se duas raízes reais iguais; $D i s c>0$ : tem-se três raízes reais;

\subsection{Método Analítico}

No método de Cardano (Cardano, 1968), a primeira etapa consiste em substituir $C_{i}$ da Equação 4 por $c_{i}=C_{i} / C_{1} \quad \operatorname{com}\left(C_{1} \neq 0\right)$. Substituindo a variável $\mathrm{Z}$ conforme a Equação 9:

$$
Z=z+\beta
$$

Escolhendo-se $\beta=-c_{2} / 3$ o termo quadrático é eliminado da equação e a função $f$ é convertida em um polinômio da seguinte forma:

$$
z^{3}+p z+q=0
$$

Definindo:

$$
p=\frac{-1}{3} c_{2}^{2}+c_{3} \quad q=\frac{2}{27} c_{2}^{3}-\frac{1}{3} c_{2} c_{3}+c_{4}
$$

O discriminante é definido da seguinte forma:

$$
\text { Disc }=\frac{p^{3}}{27}+\frac{q^{2}}{4}
$$

As possíveis soluções para $Z$ pelo método de Cardano são:

$$
\begin{aligned}
& \text { Disc } \leq 0 \quad Z_{K}=\left[2\left(-\frac{p}{3}\right)^{\frac{1}{2}} \cos \left(\frac{1}{3} \arccos \frac{-q / 2}{\sqrt{-p^{3} / 27}}\right)-\frac{c_{2}}{3}\right] k=1,2,3 \\
& \text { Disc } \leq 0 \quad Z=\sqrt[3]{-q / 2+\sqrt{D i s c}}+\sqrt[3]{-q / 2-\sqrt{D i s c}}-\frac{c_{2}}{3}
\end{aligned}
$$

Por fim, as soluções obtidas em cada um dos métodos, analítico ( $\left.Z_{\text {analítico }}\right)$ e numérico ( $Z_{\text {numérico }}$ ), foram comparadas através do Desvio Médio Relativo (DMR \%) dado por: 


$$
(D M R \%)=\frac{1}{N} \sum_{i=1}^{N}\left(\frac{Z_{\text {analítico }}-Z_{\text {numérico }}}{Z_{\text {analítico }}}\right)
$$

em que, $N$ é o número total de resultados.

\section{RESULTADOS E DISCUSSÕES}

Para o fluido analisado neste trabalho, utilizando o simulador PVTpetro, verificou-se a existência de duas fases (óleo e gás) para o intervalo de pressão de 0 - 500 bar e temperatura de $30-120^{\circ} \mathrm{C}$. A Figura 1 apresenta o comportamento do fator de compressibilidade da fase vapor $\left({ }_{V}\right)$ em função da pressão, utilizando os métodos analítico (MA) e numérico (MN).

Figura 1 - Análise do fator de compressibilidade da fase vapor com a pressão, utilizando os métodos analítico $(\mathrm{MA})$ e numérico $(\mathrm{MN})$, parametrizado em $\mathrm{T}=65,85 \mathrm{e}$ $105^{\circ} \mathrm{C}$.

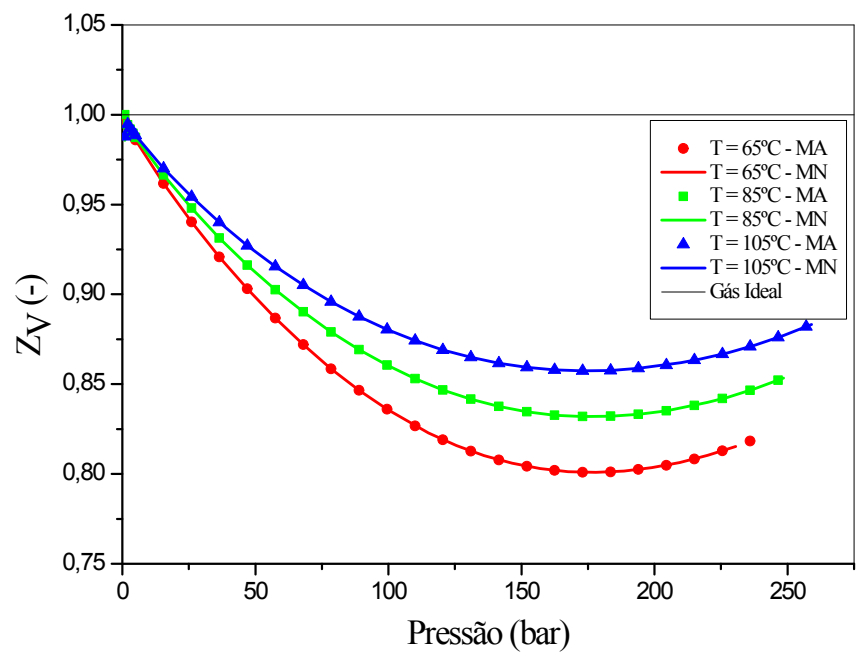

Analisando o comportamento das curvas do fator de compressibilidade da fase vapor $\left({ }_{V}\right)$ do sistema bifásico, verifica-se uma boa concordância entre os métodos de cálculo utilizados. Conforme esperado, a temperatura influencia significativamente no comportamento de $Z_{V}$ à medida que a pressão aumenta. Além disso, é possível verificar valores distintos para a pressão de bolha. Os valores observados de pressão de bolha, foram: 236 bar $\left(65^{\circ} \mathrm{C}\right), 246,5$ bar $\left(85^{\circ} \mathrm{C}\right)$ e 257 bar $\left(105^{\circ} \mathrm{C}\right)$. Do ponto de vista termodinâmico, a consideração da influência tanto da pressão como da temperatura na descrição do comportamento de fases é de fundamental importância. Pois, observa-se uma não idealidade das curvas de $Z_{V}$ quando comparadas a curva do gás ideal, à medida que a pressão afasta-se da condição ambiente (1 bar).

De maneira análoga, o fator de compressibilidade da fase líquida $\left(\mathrm{Z}_{\mathrm{L}}\right)$ do sistema óleogás multicomponente foi analisado em função da pressão em temperaturas distintas, conforme mostra a Figura 2. No caso do comportamento do $Z_{L}$, foi realizada uma comparação em relação ao comportamento de um fluido incompressível. 
Figura 2 - Análise do fator de compressibilidade da fase líquida com a pressão, utilizando os métodos analítico (MA) e numérico $(\mathrm{MN})$, parametrizado em $\mathrm{T}=65,85 \mathrm{e}$ $105^{\circ} \mathrm{C}$.



As curvas do fator de compressibilidade da fase líquida apresentadas na Figura 2 são similares para ambos os métodos de cálculo de $\mathrm{Z}$ utilizados. Avaliando o comportamento das curvas de $Z_{L}$ no intervalo de pressão de $0-500$ bar verifica-se a formação de duas regiões distintas. Na primeira região $(0-260$ bar $)$ tem-se as fases óleo e gás em equilíbrio. Nesta região, o $Z_{L}$ sofre forte influência da pressão devido ao processo de solubilização do gás na fase óleo ocasionando um desvio do comportamento ideal. A temperatura apresenta pouca influência no comportamento de $Z_{L}$ nesta região bifásica. A segunda região $(260-500$ bar $)$ contém apenas a fase líquida devido a total solubilização do gás no óleo. Nesta região monofásica, tanto a pressão como a temperatura apresentam pequena influência no comportamento das curvas, as quais apresentam um comportamento semelhante ao de um líquido incompressível.

Na Tabela 1, os desvios médios relativos gerados na comparação dos valores dos fatores de compressibilidades das fases em equilíbrio são apresentados para as temperaturas de 65,85 e $105^{\circ} \mathrm{C}$.

Tabela 1 - Comparação dos métodos de cálculo do fator de compressibilidades das fases.

\begin{tabular}{cccc}
\hline \multirow{2}{*}{$\operatorname{DMR}(\%)$} & \multicolumn{3}{c}{ Temperatura $\left({ }^{\circ} \mathrm{C}\right)$} \\
\cline { 2 - 4 } & 65 & 85 & 105 \\
\hline$Z_{\mathrm{V}}$ & $9,8708 \mathrm{E}-02$ & $7,4990 \mathrm{E}-02$ & $2,3123 \mathrm{E}-02$ \\
\hline$Z_{\mathrm{L}}$ & $9,3569 \mathrm{E}-01$ & $1,5008 \mathrm{E}+00$ & $2,4164 \mathrm{E}-01$ \\
\hline
\end{tabular}

A partir dos valores apresentados na Tabela 1, verificam-se pequenos desvios entres os resultados gerados pelos métodos analítico e numérico para o fator de compressibilidade das fases líquida e vapor. 


\section{CONCLUSÕES}

Diante dos resultados apresentados e do objetivo proposto neste trabalho conclui-se que para o cálculo do fator de compressibilidade das fases de um sistema bifásico, o método analítico e o método numérico apresentaram resultados semelhantes com valores de DMR menor que $2 \%$. Além disso, verificou-se que as variáveis pressão e temperatura influenciaram fortemente na determinação de $Z_{V}$, mostrando uma não idealidade no comportamento desta variável. Em relação ao $Z_{L}$, verificou-se a existência de duas regiões, para as condições investigadas, com comportamentos distintos.

\section{REFERÊNCIAS}

AHMED, T. Equations of State and PVT Analysis: Applications for Improved Reservoir Modeling. Texas, USA: Gulf Publishing Company, 2007.

ANP. Boletim da Produção de Petróleo e Gás Natural. Superintendência de Desenvolvimento e Produção - SDP, 2014.

BARBOSA NETO, A. M. Desenvolvimento de um simulador PVT composicional para fluidos de petróleo. Dissertação de Mestrado - Faculdade de Engenharia Mecânica, Departamento de Energia - Divisão de Petróleo, Unicamp, Campinas.

BARBOSA NETO, A. M.; RIBEIRO, J. AZNAR, M.; BANNWART, A. C. Thermodynamic modeling of vapor-liquid Equilibrium for Petroleum fluids. $1^{\circ}$ CNMAI, 2014.

CARDANO, G. Ars Magna, The rules of Algebra. New York : Dover Publications, 1968.

PENG, D. Y.; ROBINSON, D. B. The characterization of the heptanes and heavier fractions for the GPA Peng-Robinson programs. Research Report RR-28, Gas Processors Association., 1978.

SABER, N. Phase behaviour prediction for Ill-defined hydrocarbon mixtures. 2011. 175p. Thesis (Doctorate) - Department of Chemical and Materials Engineering, University of Alberta, Edmonton.

SABER, N.; SHAW, J. M. Rapid and robust phase behaviour stability analysis using global optimization. Fluid Phase Equilibria, v. 264, p.137-146, 2008. 